\title{
Neurodynamics of up and down Transitions in Network Model
}

\author{
Xuying $X u^{1}$ and Rubin Wang ${ }^{2}$ \\ ${ }^{1}$ Institute for Cognitive Neurodynamics, School of Science, East China University of Science and Technology, Shanghai 200237, China \\ ${ }^{2}$ Institute for Cognitive Neurodynamics, School of Science, School of Information Science and Engineering, \\ East China University of Science and Technology, Shanghai 200237, China
}

Correspondence should be addressed to Rubin Wang; rbwang@163.com

Received 20 November 2013; Accepted 27 November 2013

Academic Editor: Jinde Cao

Copyright (C) $2013 \mathrm{X}$. Xu and R. Wang. This is an open access article distributed under the Creative Commons Attribution License, which permits unrestricted use, distribution, and reproduction in any medium, provided the original work is properly cited.

\begin{abstract}
This paper focuses on the neurodynamical research of a small neural network that consists of 25 neurons. We study the periodic spontaneous activity and transitions between up and down states without synaptic input. The results demonstrate that these transitions are bidirectional or unidirectional with the parameters changing, which not only reveals the function of the cortex, but also cohere with the experiment results.
\end{abstract}

\section{Introduction}

Different patterns of brain activity can give rise to different behavioral states of the animals. Neural electrophysiology experiments show that during slow-wave sleep in the primary visual cortex of anesthetized animals [1-3] and during quiet wakefulness in the somatosensory cortex of unanesthetized animals $[4,5]$, the membrane potentials make spontaneous transitions between two different levels called up and down states [6]. Transitions between up and down states can also be evoked by sensory stimulation [1, 4, 7-11]. An interesting result of these transitions is that sensory-evoked activity patterns are similar to those produced spontaneously. A hallmark of this subthreshold activity is a bimodal distribution of the membrane potential [12]. However, why these transitions occur or whether this spontaneous activity engages in brain functions or not remains unclear. In fact, we know little about expressions of neuron membrane potentials and interactions between neural networks, especially the relationship between neural coding modes and cognitive behaviors. So our purpose is to try to understand the inner connection between the up and down transitions of a single neuron and that of neural network.

Recent findings show that activation of a single cortical neuron can significantly modulate sensory and motor outputs $[13,14]$. Furthermore, repetitive high frequency burst spiking of a single rat cortical neuron could trigger a switch between the cortical states resembling slow-wave and rapideye-movement sleep [15]. This is reflected in the switching of membrane potential of the stimulated neuron from high frequency and low amplitude oscillations to low frequency and high amplitude ones or vice versa. At the same time, cortical local field potential (LFP) changes over time. Here we use local field potential (LFP) to describe the state of the whole cortex [16-19]. Therefore, the up and down states of single neuron reflect distinct global cortical states, which resemble slow-wave and rapid-eye-movement sleep, respectively [20-22]. All of these results point to the power of single cortical neurons in modulating the behavior state of animals [15]. Here, one single neuron affects the whole network status by impacting other coupling neurons.

We have started our research on a single neuron, studied the electrophysiological phenomenon of status transitions, and obtained the bistability and spontaneity that is similar to experiment observation. In addition, we found that these up and down transitions show unidirectional or bidirectional changing with different parameters. Bistability means that the neuron stays in one state before stimulation and turn to another state after stimulation. These two states are called up state and down state, respectively. That is to say, the neuron can switch between up and down states. And directivity refers to the fact that it is not arbitrary to switch from one state to another. In some cases, transition can only occur from up state to down state, while it occurs from down state to up state 
in other cases. Spontaneity, the periodic spontaneous activity of neural membrane potential, is the most significant feature of the transition.

This paper tries to further explore neural dynamic mechanism of up and down transitions in a neural network based on the above results. This work will lay a foundation for studying the relationship between neural coding and cognitive behavior. We focus on the dynamic process of the average membrane potential of a small neural network that consists of 25 neurons and switches between up and down states. And we observe the difference and distinguish between transitions in network and one single neuron by numerical simulation and theoretical analysis. Then we try to know what happened to the appearance of behavior states and the inducement of brain cognition. What we want to highlight is that how great the effect of the emerged relationship between behavior states and cognition and the ratio of activated neurons on up and down transitions is. This is also the subject for our further study.

\section{Network Model}

There are different kinds of complicated connection between neurons. According to the topology, some scholars proposed chain link, ring link, grid link, and so forth [23]. However, the internal connections between neurons are much more complicated than those above connections. This paper constructs a dynamical network model that consists of 25 neurons based on previous study [24]. In this network, any one neuron connects to any other neurons in the network. That means every two neurons in the network are coupled with the connection strength asymmetrically and obeying standard uniform distribution [25].

The coupling strength between neurons can be expressed with matrix variable, denoted by $G$. Then we have

$$
G=\left[\begin{array}{cccc}
g_{1,1} & g_{1,2} & \ldots & g_{1, n} \\
g_{2,1} & \ddots & & g_{2, n} \\
\vdots & & \ddots & \vdots \\
g_{n, 1} & g_{n, 2} & \ldots & g_{n, n}
\end{array}\right]=\left[\begin{array}{cccc}
0 & g_{1,2} & \ldots & g_{1, n} \\
g_{2,1} & 0 & \cdots & g_{2, n} \\
\vdots & \vdots & \ddots & \vdots \\
g_{n, 1} & g_{n, 2} & \ldots & 0
\end{array}\right]
$$

where $g_{i, j}$ represents the coupling strength form neuron $i$ to neuron $j$. Absolutely, neurons do not couple with themselves, so these coupling strength denoted by $g_{i, j}$ equals to zero.

To illustrate the state changing of the whole network, we use the changing of average membrane potential to describe the changing of local field potential (LFP), which means that we average membrane potentials of every neuron in the network to express LFP.

The dynamic model of one neuron in the neural network is based on $\mathrm{H}-\mathrm{H}$ equations and is described by (2) to (8). This dynamic model consists of three ionic currents and synaptic currents which come from surrounding neurons. The ionic currents contain an instantaneous, inward current (sodium current), a slow $h$-like current $[26,27]$, and an outward current (a potassium current and a leak current). Two types of persistent inward, persistent sodium, and persistent calcium have been characterized in Purkinje cells [28, 29]. Somatic
Purkinje cell bistability has been associated with persistent sodium whereas dendritic bistability has been shown to result from persistent calcium conductance. Here we use persistent sodium in our model for simplicity but it is likely that it is the combination of these two currents that enables the bistability [30].

On the basis of previous research, we propose the following neural network model to study the characteristics, bistability, directivity, and spontaneity, of the up and down transitions that have been observed in electrophysiology experiments. Thus, we clarify the neural dynamic mechanism of the up and down transitions in neural network. The current equation for the model is

$$
C \frac{d V_{i}}{d t}=-\left(I_{\mathrm{Na}}^{i}+I_{h}^{i}+I_{K}^{i}+I_{l}^{i}+I_{\mathrm{syn}}^{i}\right) .
$$

Here, the ionic currents are as follows:

$$
\begin{gathered}
I_{\mathrm{Na}}^{i}=g_{\mathrm{Na}} m_{\infty}\left(V_{i}-V_{\mathrm{Na}}\right), \\
I_{h}^{i}=g_{h} h_{i}\left(V_{i}-V_{h}\right), \\
I_{K}^{i}=g_{K} b_{i}\left(V_{i}-V_{K}\right), \\
I_{l}^{i}=g_{l}\left(V_{i}-V_{l}\right),
\end{gathered}
$$

where

$$
m_{\infty}=\left(1+e^{-\left(V_{i}-T_{m}\right) / \sigma_{m}}\right)^{-1} .
$$

The synaptic current is

$$
I_{\text {syn }}^{i}=\sum_{j \neq i} g_{j i}\left(V_{i}-V_{j}\right)
$$

The synaptic current of the $i$ th neuron is a sum of effects from all the other neurons in the network, so this kind of current plays a key role in coupling every two neurons in the whole network. The changing activity of one neuron affects the whole network states changing through this way.

There are two dynamic variables: membrane potential $V_{i}$ and the inactivation term of the $h$-current $h_{i}$, when we discuss the bistability and directivity. But when studying the spontaneity, we need another variable called the inactivation term of potassium current $b_{i}$. The dynamics of the inactivation terms of $h$-current and potassium current are

$$
\begin{aligned}
\frac{d h_{i}}{d t} & =\frac{h_{\infty}-h_{i}}{\tau_{h}}, \\
\frac{d b_{i}}{d t} & =\frac{b_{\infty}-b_{i}}{\tau_{b}},
\end{aligned}
$$


where

$$
\begin{gathered}
h_{\infty}=\left(1+e^{\left(V_{i}-T_{h}\right) / \sigma_{h}}\right)^{-1}, \\
\tau_{h}=\frac{1}{\alpha+\beta}, \\
\alpha=\frac{a_{\alpha} \cdot V_{i}+b_{\alpha}}{1-e^{\left(\left(V_{i}+b_{\alpha}\right) / a_{\alpha}\right) / k_{\alpha}}}, \\
\beta=\frac{a_{\beta} \cdot V_{i}+b_{\beta}}{1-e^{\left(\left(V_{i}+b_{\beta}\right) / a_{\beta}\right) / k_{\beta}},} \\
b_{\infty}=\left(1+e^{-\left(V_{i}-T_{b}\right) / \sigma_{b}}\right)^{-1}, \\
\tau_{b}=\tau_{b}^{0} \cdot \sec \left(h_{i} \frac{V_{i}-T_{b}}{4 \cdot \sigma_{b}}\right) .
\end{gathered}
$$

In these equations, $V_{i}$ represents membrane potential of the $i$ th neuron, while $I_{h}^{i}, I_{K}^{i}, I_{l}^{i}$ replace a sodium current, a slow $h$-like current, and a potassium current and a leak current of the $i$ th neuron, respectively. Similarly, $g_{\mathrm{Na}}, g_{h}$, $g_{K}, g_{l}$, respectively, represent the sodium conductance, the slow $h$-like conductance, the potassium conductance, and the leak conductance, and $V_{\mathrm{Na}}, V_{h}, V_{K}, V_{l}$ are the corresponding reversal potentials. The inactivation term of the sodium current, the $h$-like current, and the potassium current are described by $m_{\infty}, h_{\infty}, b_{\infty}$, and dynamic variables $h_{i}, b_{i}$, with the synaptic time constant $\tau_{h}, \tau_{b}$. And $T_{m}, \sigma_{m}, T_{h}, \sigma_{h}, T_{b}, \sigma_{b}$, $\tau_{b}^{0}, a_{\alpha}, b_{\alpha}, k_{\alpha}, a_{\beta}, b_{\beta}, k_{\beta}$ are constants.

\section{Results}

3.1. Bistability. When we studied the single neuron model, we found that transitions between up and down states can be induced by two different kinds of stimulus. One is to add brief outward current pulses; another is to improve the sodium conductance to a certain value instantaneously. Now, we research the neural network in the same way to try to find out that there exist the similar phenomenon or not which agrees with electrophysiology experiment results.

In the period of 10 seconds, we add a pulse current which lasts 0.1 second every two seconds, with the current intensity $7.2 \mu \mathrm{A} / \mathrm{cm}^{2}$. The results are shown in Figure 1. We find that the average membrane potential switches between the up state (about $-45 \mathrm{mV}$ ) and the down state (about $-65 \mathrm{mV}$ ).

In the period of 10 seconds, we add the stimulation that lasts $4 \mathrm{~ms}$ every one or two seconds, leading to the intensity of sodium conductance changing from $0.06 \mathrm{mS} / \mathrm{cm}^{2}$ to $1.2 \mathrm{mS} / \mathrm{cm}^{2}$ instantaneously. The results are shown in Figure 2. We find that the average membrane potential switches between the up state (about $-45 \mathrm{mV}$ ) and the down state (about $-65 \mathrm{mV}$ ) when adding the same stimulation. And these transitions are a little bit complex: the membrane potential rises up to $0 \mathrm{mV}$ instantaneously but then drops quickly.

So from the above two results, we find that this dynamic model can describe the bistability of up and down transitions of neural membrane potential in the neural network. That

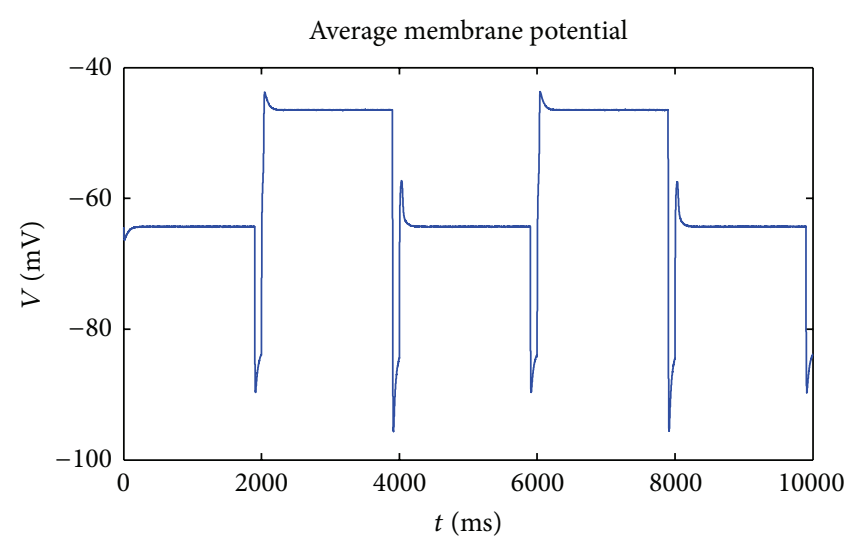

FIGURE 1: Add brief outward current pulses (lasts $0.1 \mathrm{~s}$, every $2 \mathrm{~s}$, $\left.7.2 \mu \mathrm{A} / \mathrm{cm}^{2}\right)$.

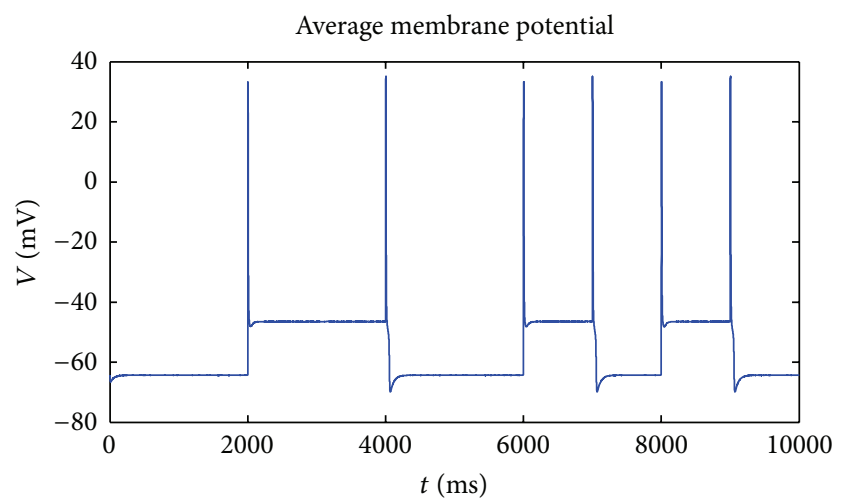

FIGURE 2: Increase the sodium conductance instantaneously $\left(0.06 \mathrm{mS} / \mathrm{cm}^{2}\right.$ to $\left.1.2 \mathrm{mS} / \mathrm{cm}^{2}\right)$.

means there are two stable states for neural network, with other states unstable. The network can stay in one of these two stable states without any input. When the neuron in the network is stimulated, which destroys its original stability, it can switch its state from one to another to adjust itself to a new balance. These two states are called the up state and the down state, respectively. That is to say, the up and down transitions can be modulated by external stimulations. The ionic movement between inside and outside of the membrane may be the mechanism of the transitions. When sodium conductance increases to a certain level, it causes slight depolarization, activating the sodium channel with sodium move into cells, which increases the range of the polarization. In return, the larger the range of depolarization occurs, the more the sodium channels are activated and the more the sodium moves into cells. When it arrives to the peak of membrane potential, the sodium channel is inactivated and the $h$-like channel is activated, which leads to repolarization of the membrane potential. When the membrane potential reduces to about $-45 \mathrm{mV}, h$-like channel is inactivated. At this point, a new balance between the outflow of potassium and the inflow of sodium begins. Namely, membrane potential stays in a stable state. According to the different extent of the 


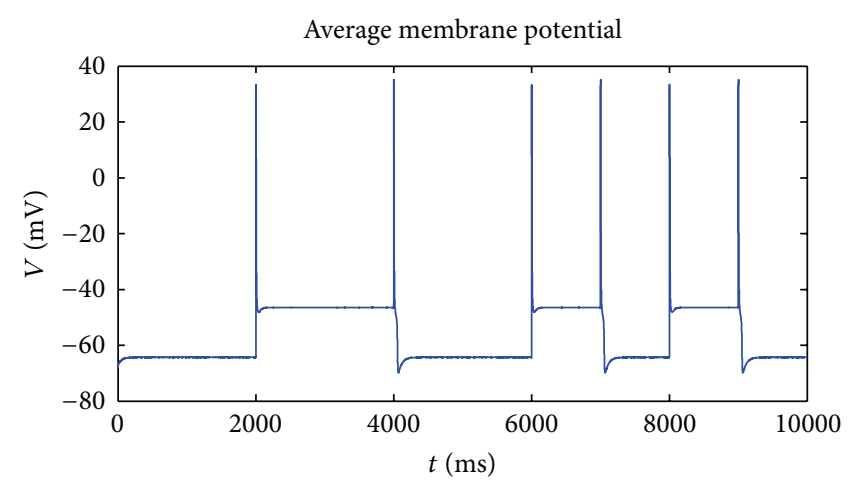

(a)

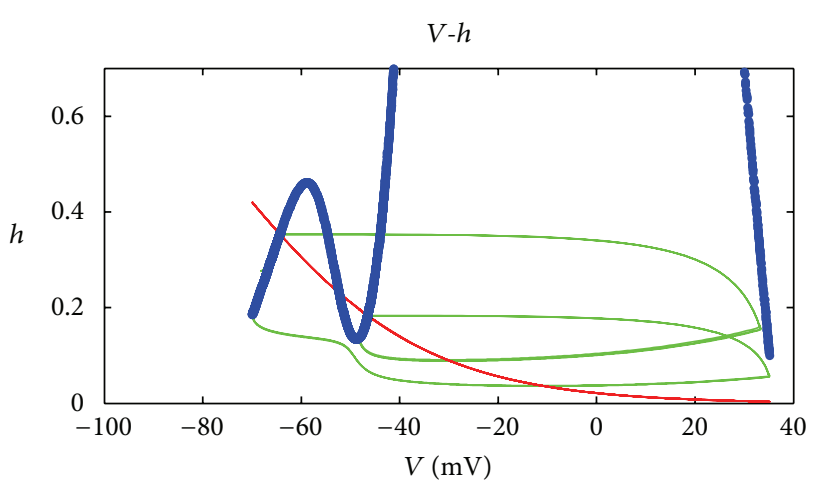

(b)

FIgURE 3: Average membrane potential $V$ and $h-V$ phase plane when $g_{k}=0.1 \mathrm{mS} / \mathrm{cm}^{2}$ and $g_{\mathrm{Na}}=1.2 \mathrm{mS} / \mathrm{cm}^{2}$.

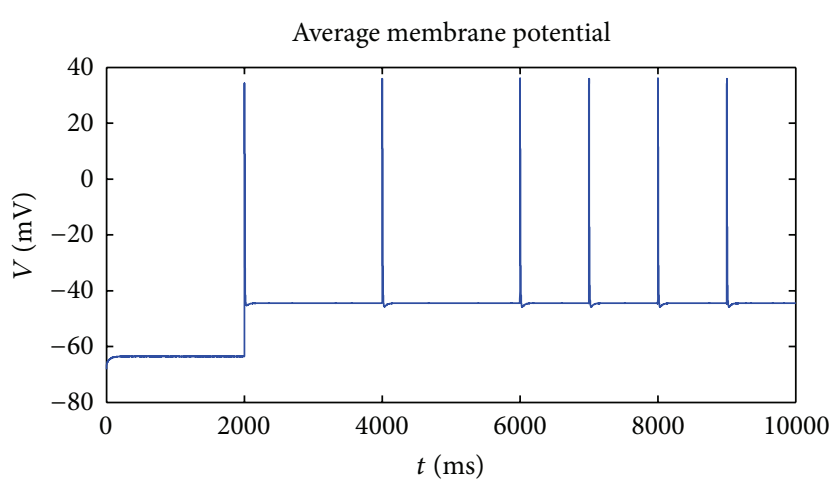

(a)

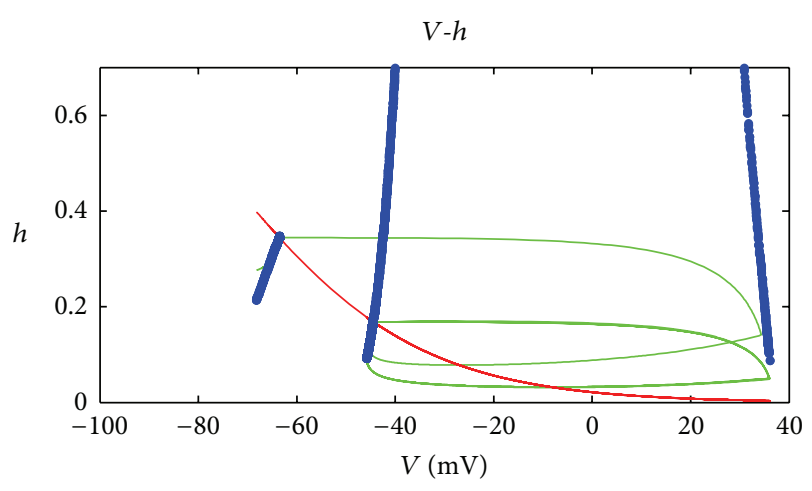

(b)

FIGURE 4: Average membrane potential $V$ and $h-V$ phase plane when $g_{k}=0.09 \mathrm{mS} / \mathrm{cm}^{2}$ and $g_{\mathrm{Na}}=1.2 \mathrm{mS} / \mathrm{cm}^{2}$.

$h$-like channel inhibition, the membrane potential stays in the up state (about $-45 \mathrm{mV}$ ) or the down state (about $-65 \mathrm{mV}$ ).

3.2. Directivity. In the model of a single neuron, directivity of the transition is modulated by potassium conductance. We found that when $g_{k}=0.1 \mathrm{mS} / \mathrm{cm}^{2}$, membrane potential can transit both from the down state to the up state and from the up state to the down state, when $g_{k}=0.09 \mathrm{mS} / \mathrm{cm}^{2}$, membrane potential can only transit from the down state to the up state, and when $g_{k}=0.105 \mathrm{mS} / \mathrm{cm}^{2}$, membrane potential can only transit from the up state to the down state.

In the model of neural network of this paper, we also do research on the directivity. And we find that the changing of sodium conductance can modulate the directivity of the transitions as well as potassium conductance. Figures 37 describe different transition modes adjusted by different values of potassium conductance and sodium conductance. The tops of Figures 3-7 are average membrane potential $V$ of the neural network, namely, up and down transitions, while the bottoms are phase plane for the mean of two kinds of dynamic variables $h$ and $V$ in the model, denoted by $V_{\text {mean }}, h_{\text {mean }} . V_{\text {mean }}$ is average membrane potential of all the neurons in the network. $h_{\text {mean }}$ is average inactivation rate of all the $h$-like channel in the network. The red solid line shows all the points that $h^{\prime}=0$, the blue dot line shows all the points that $V^{\prime}=0$, and the intersection of these two lines is stable point of the system. In other words, the two points are stable states of the network, and other points in the plane are unstable. That means, the system will stay in any one of the two stable points after a long run. The green solid line in the figure presents the transit process from one stable point to another.

Figure 3 shows that when $g_{k}=0.1 \mathrm{mS} / \mathrm{cm}^{2}$, membrane potential can transit from the down state to the up state by adding a stimulation that increase sodium conductance to $g_{\mathrm{Na}}=1.2 \mathrm{mS} / \mathrm{cm}^{2}$ instantaneously. With the same stimulation, it also can transit from the up state to the down state. So the transitions are bidirectional on condition that $g_{k}=0.1 \mathrm{mS} / \mathrm{cm}^{2}$ and $g_{\mathrm{Na}}=1.2 \mathrm{mS} / \mathrm{cm}^{2}$. The $h-V$ phase plane further shows that the system transmits between the two stable states.

We can observe the changing of up and down transitions of the whole network by making some changes on the potassium conductance while keeping sodium conductance unchanged; namely, $g_{\mathrm{Na}}=1.2 \mathrm{mS} / \mathrm{cm}^{2}$. The results are shown in Figures 4-5.

Figure 4 represents that when $g_{k}=0.09 \mathrm{mS} / \mathrm{cm}^{2}$, the average membrane potential can transit from the down state 


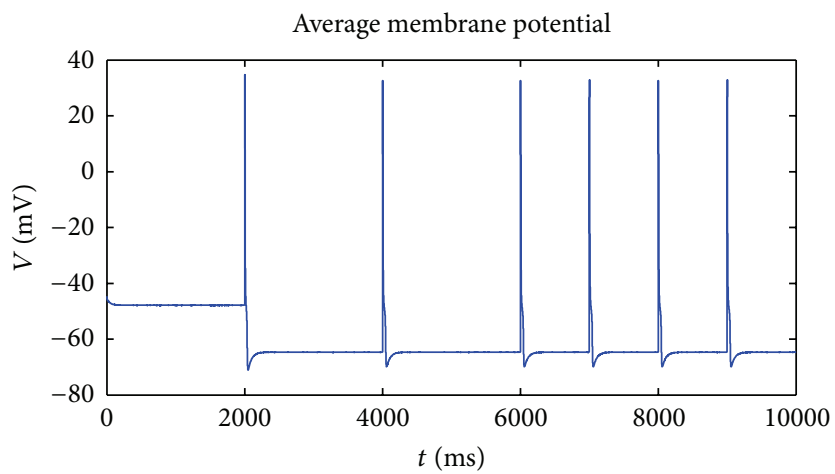

(a)

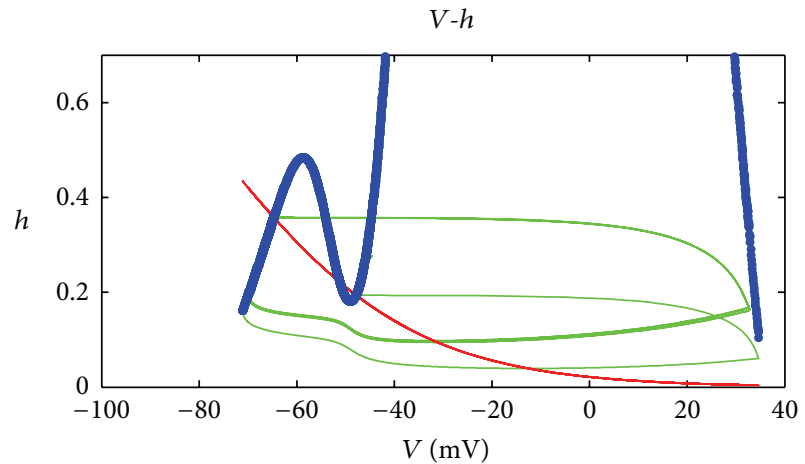

(b)

FIGURE 5: Average membrane potential $V$ and $h-V$ phase plane when $g_{k}=0.105 \mathrm{mS} / \mathrm{cm}^{2}$ and $g_{\mathrm{Na}}=1.2 \mathrm{mS} / \mathrm{cm}^{2}$.

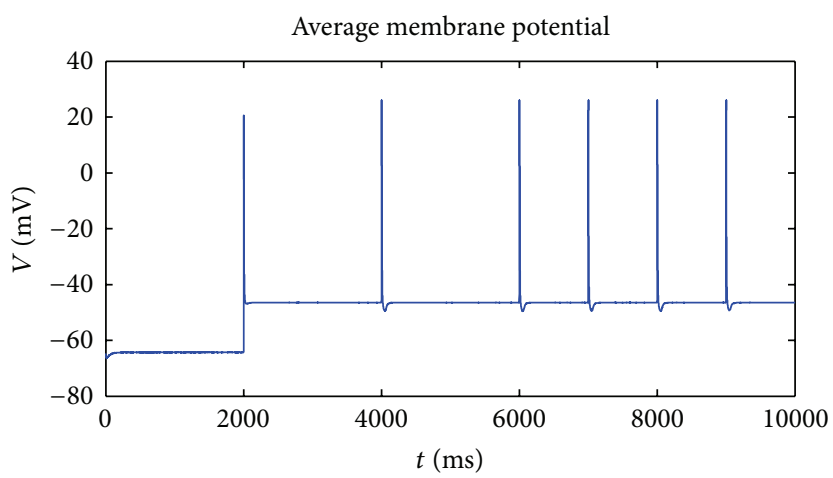

(a)

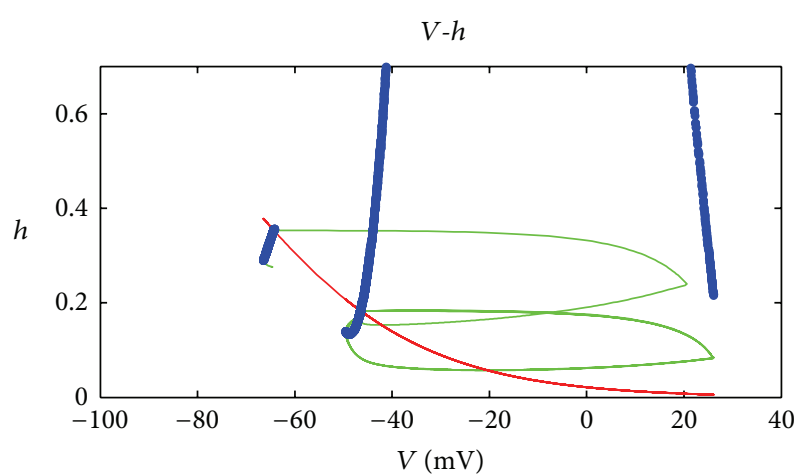

(b)

FIGURE 6: Average membrane potential $V$ and $h-V$ phase plane when $g_{k}=0.1 \mathrm{mS} / \mathrm{cm}^{2}$ and $g_{\mathrm{Na}}=0.8 \mathrm{mS} / \mathrm{cm}^{2}$.

to the up state by adding a stimulation that increase sodium conductance instantaneously. But with the same stimulation, the average membrane potential always stays in the up state without any change. In other words, the transitions that are unidirectional vary from the down state to the up state in the circumstances that $g_{k}=0.09 \mathrm{mS} / \mathrm{cm}^{2}$. The $h-V$ phase plane also shows that the system can only vary from the lower membrane potential stable point to higher one and then move around the higher one periodically.

Figure 5 reveals that when $g_{k}=0.105 \mathrm{mS} / \mathrm{cm}^{2}$, the average membrane potential can transit from the up state to the down state by adding a stimulation that increase sodium conductance instantaneously. However, with the same stimulation, the average membrane potential always stays in the down state without any change. In other words, the transitions that are unidirectional vary from the up state to the down state under the circumstances that $g_{k}=$ $0.105 \mathrm{mS} / \mathrm{cm}^{2}$. The $h-V$ phase plane also presents that the system can only vary from the higher membrane potential stable point to lower one and then move around the lower one periodically.

Accordingly, we can also observe the changing of up and down transitions of the whole network by making some changes on the sodium conductance while keeping potassium conductance unchanged; namely, $g_{k}=0.1 \mathrm{mS} / \mathrm{cm}^{2}$. The results are shown in Figures 6-7.

Figure 6 presents that when $g_{\mathrm{Na}}=0.8 \mathrm{mS} / \mathrm{cm}^{2}$, the average membrane potential can transit from the down state to the up state by adding a stimulation that increase sodium conductance instantaneously. But with the same stimulation, the average membrane potential always stays in the up state without any change. In other words, the transitions that are unidirectional vary from the down state to the up state in the circumstances that $g_{\mathrm{Na}}=0.8 \mathrm{mS} / \mathrm{cm}^{2}$. The $h-V$ phase plane also shows that the system can only vary from the lower membrane potential stable point to higher one and then move around the higher one periodically.

Figure 7 reveals that when $g_{\mathrm{Na}}=2 \mathrm{mS} / \mathrm{cm}^{2}$, the average membrane potential can transit from the up state to the down state by adding a stimulation that increase sodium conductance instantaneously. However, with the same stimulation, the average membrane potential always stays in the down state without any change. In other words, the transitions that are unidirectional vary from the up state to the down state under the circumstances that $g_{\mathrm{Na}}=2 \mathrm{mS} / \mathrm{cm}^{2}$. The $h-V$ phase plane also presents that the system can only vary from the higher membrane potential stable point to lower one and then move around the lower one periodically. 


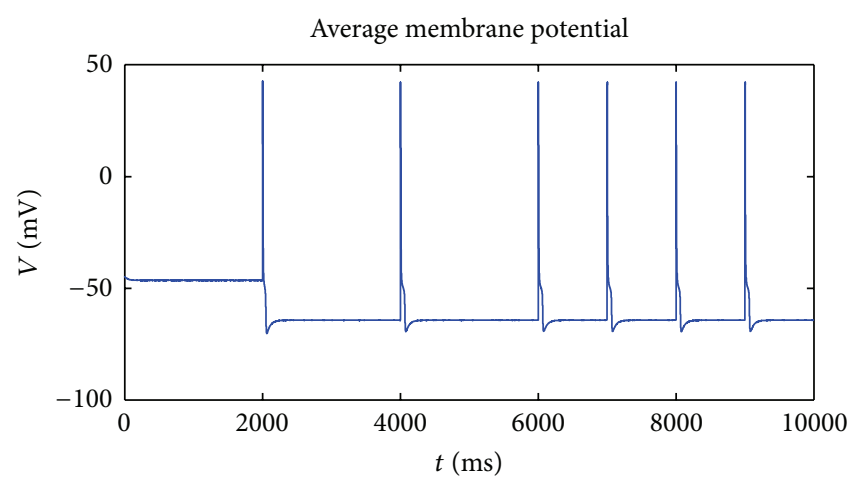

(a)

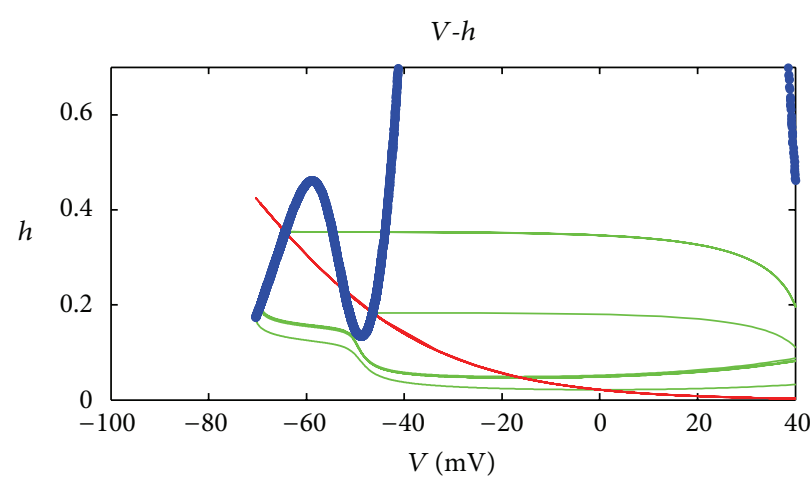

(b)

Figure 7: Average membrane potential $V$ and $h-V$ phase plane when $g_{k}=0.1 \mathrm{mS} / \mathrm{cm}^{2}$ and $g_{\mathrm{Na}}=2 \mathrm{mS} / \mathrm{cm}^{2}$.

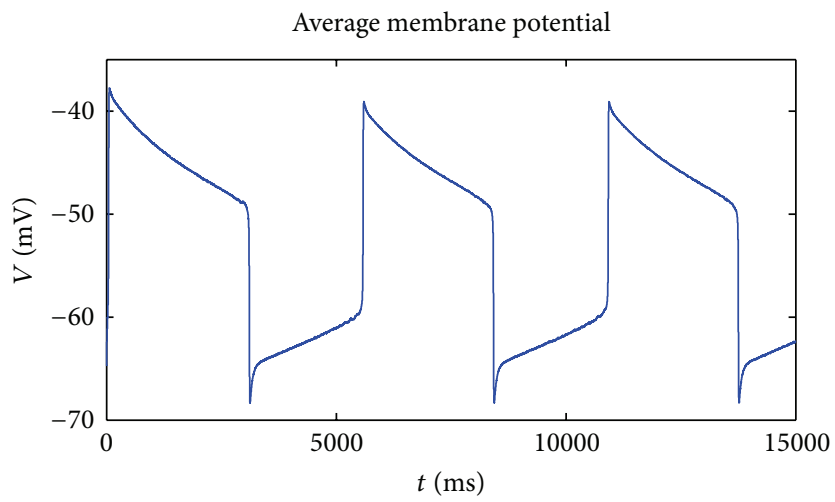

(a)

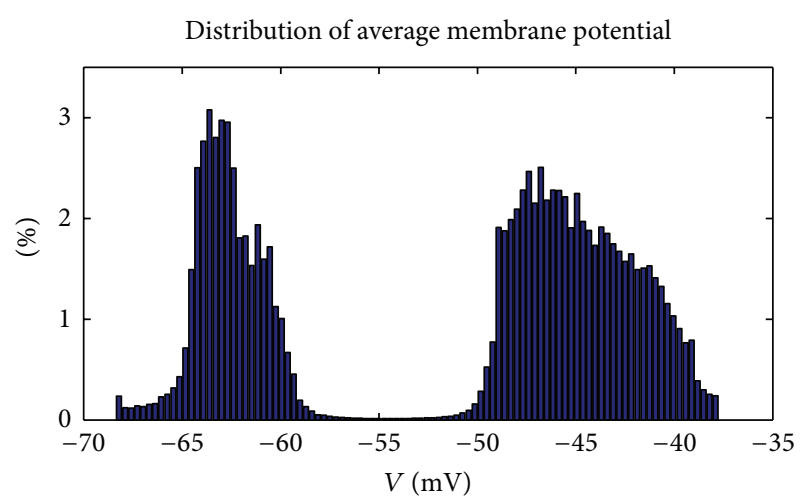

(b)

FIGURE 8: The average membrane potential of spontaneous transitions and its distribution.

The above results reveal that this dynamic model can well describe the bidirectional or unidirectional characteristic of up and down transitions of neural network when stimulated by certain stimulus. These results accord with the results of a single neuron model. Transitions in the network can be bidirection from the up state to the down one and vice versa. And also may be single direction from the up state to the down one, or only from down state to the up one according to the different level of conductance.

3.3. Spontaneity. In the discussion of bistability and direction of the model of a single neuron, we should introduce the input of synapse to generate the up and down transitions. Is a neuron still able to up and down transit, if there is no input of synapse? Virtually, in vivo or in vitro experiments of animals show that the potential of neural membrane can transit between up state and down state spontaneously and periodically. By increasing the variable of the inactivation of a potassium conductance rate in the original model, we can obtain the result that is identical to the experimental result.

In this paper, we introduce the dynamic variable $b$, the inactivation rate of potassium conductance of each neuron, to study the spontaneous transitions of neural networks.
The calculated results shown in Figure 8, are case without external stimuli showing that the average membrane potential transit spontaneously and periodically, while the distribution graph illustrates the distribution of the average membrane potential, a two-peak distribution, indicating the two stable state of up and down transitions of membrane potential.

By adding the interval of 1 or 2 seconds and lasting time of $4 \mathrm{~ms}$ stimuli on this spontaneous network model, the intensity of sodium conductance increases rapidly from $0.06 \mathrm{mS} / \mathrm{cm}^{2}$ to $1.2 \mathrm{mS} / \mathrm{cm}^{2}$; the computed results are shown in Figures 9-11.

The tops of Figures 9(a) and 10(a) show the changes of membrane potentials after adding stimuli, respectively, and the bottoms show the corresponding distributions of membrane potentials.

The simulating results of each neuron stimulated in the network are shown in Figure 9. Compared with the case without stimuli, after adding stimuli, the spontaneous transition of the whole network stops. Because of the disruption brought by the outer stimuli, the neurons, which should have been able to transit or transfer to another stable state 


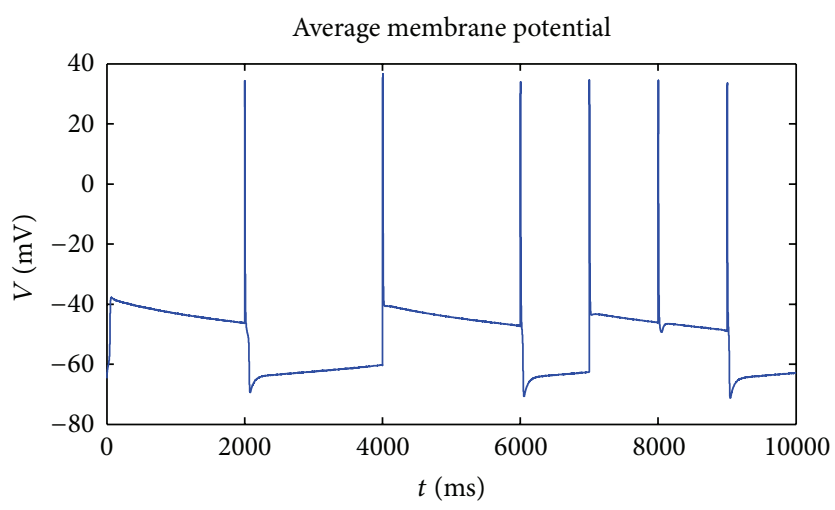

(a)

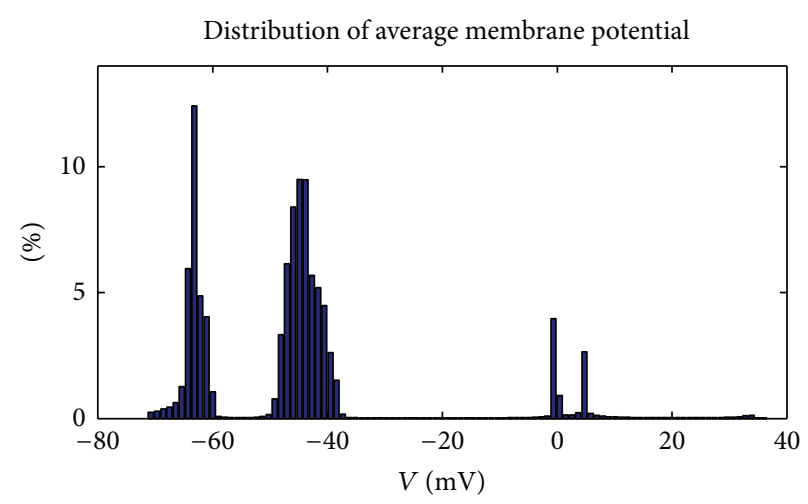

(b)

FIGURE 9: Adding stimuli on each neuron of the spontaneous model to rapidly increase the sodium conductance.

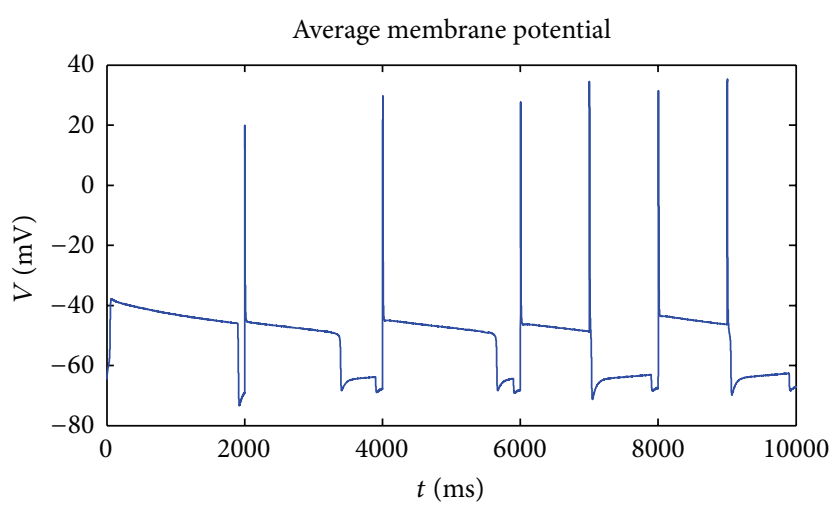

(a)

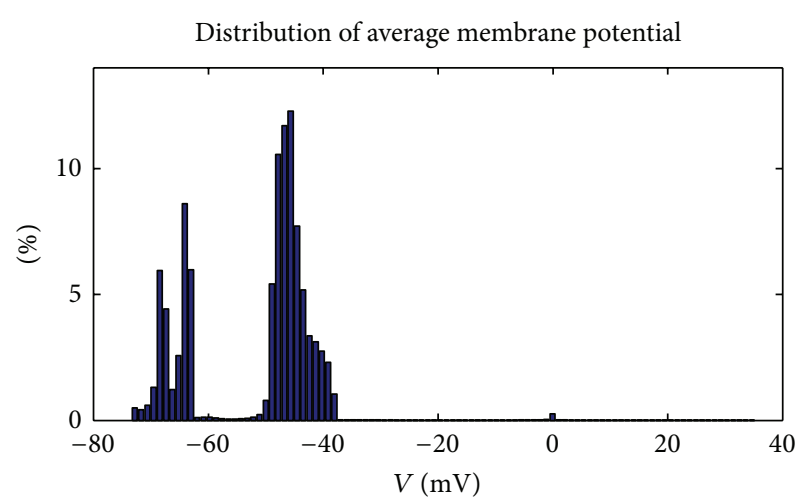

(b)

FIGURE 10: Adding stimuli on some neurons of the spontaneous model to rapidly increase the sodium conductance.

or to the original stable state of itself. However, for the whole network, the instant time for transition is different for each neuron, so the ability of network transition is not well obtained just by averaging the membrane potential. As a result, all the transitions we could see are caused by external stimuli, on the contrary, not all the stimuli can generate transition. For instance, the stimulus at $8 \mathrm{~s}$ fails to generate the state transition, which indicates that these transitions have something to do with the state of neural network. The network in a down state is more susceptible to external stimuli and transits, while in the up state it is not susceptible to external stimuli, since the network has a strong self-stability.

The simulating results of some neurons stimulated in the network are shown in Figure 10. Compared with the results in Figure 9, this network maintains its spontaneous transition ability; that is to say, it transits at the moment without any stimulus. On the other hand, the input of synapse is also able to let the state of the network transit and each stimulus leads to a state transition. Because there is coupling among the neurons, when a neuron is stimulated, such stimulus is certainly transmitted to everywhere else in the network by coupling, so as to change the state of the whole network.
To observe this transmission, Figure 11 shows the membrane potential of a neuron that is directly and indirectly stimulated, respectively. The results of the their potential are similar, since when a neuron is directly stimulated, its membrane potential changes correspondingly, and according to (8), this change transmits to others without delay. This is one of the aspects for our further improvement in the future.

The obtained results illustrate that this dynamical model dose can depict the phenomenon of the spontaneous and periodical transitions. By adjusting the number of the stimulated neurons, the situation of transition of a network differs. When every neuron is stimulated, the spontaneous transition of a network disappears, and the external stimuli play an important role on transitions. When a stimulus is added to a single neuron, besides the spontaneous transitions, the network is also able to respond to the external stimuli and transit. Such transmission between the coupling neurons is very fast and has no delay.

\section{Conclusion}

This paper constructs a dynamical network model that consists of 25 neurons which can show the up and down 


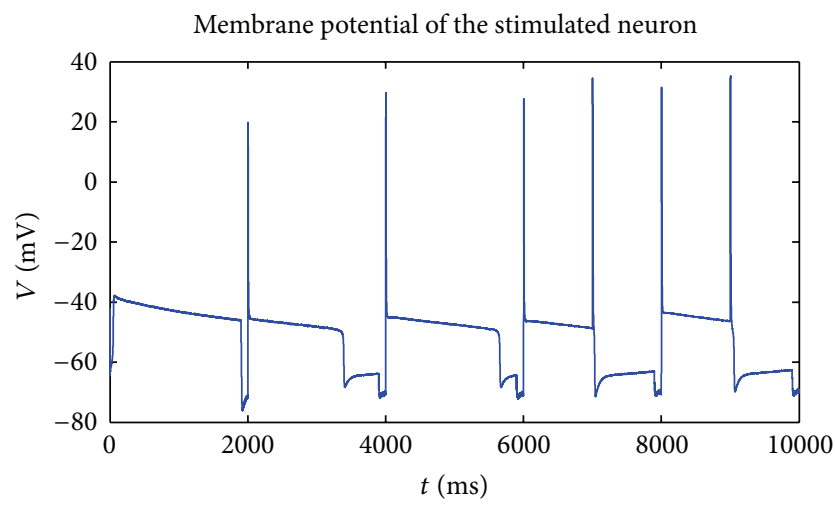

(a)

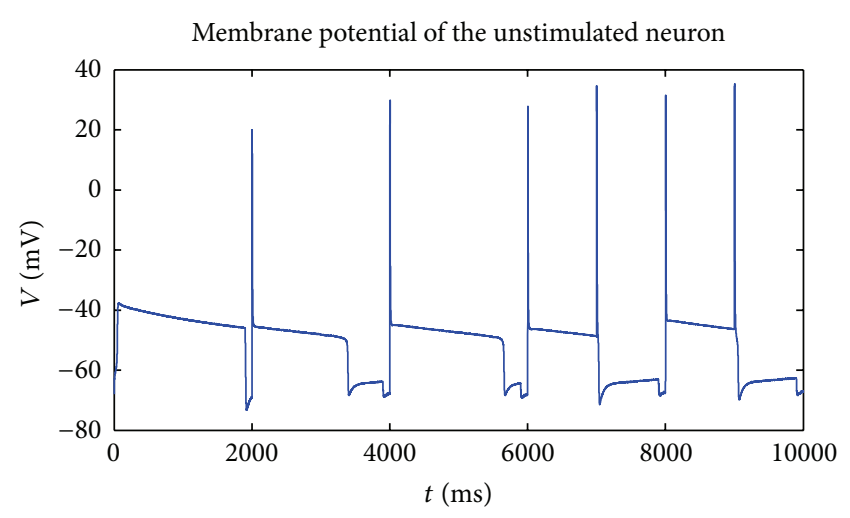

(b)

FIGURE 11: Membrane potential of a neuron is stimulated directly or indirectly, respectively.

transitions, describing three characteristics, bistability, directivity, and spontaneity, of up and down transitions. The main conclusions are as follows.

(1) This dynamic model can describe the bistalility of up and down transitions of the neural network modulated by external stimulations and sodium conductance.

(2) The dynamic model can describe the bidirectional or unidirectional characteristic of up and down transitions of the neural network controlled by potassium conductance and potassium conductance.

(3) The dynamic model can describe periodic spontaneous transitions between the up and down states in absence of input and transitions will become complex when adding synaptic input.

The above conclusions are similar to the results of up and down transitions of a single neuron, since the characteristic of a single neuron's bursting dominates the real activities of the neural networks and the dynamic of a single neuron represents the behavior of the whole networks. In this paper, the study of up and down transitions is proposed as a preparation for the further scope of large-scale neural population and up and down transitions of network behaviors, so as to understand the effect of a single neuron's transitions on network behaviors under the condition of coupling of neural population as a sort of foundation of the research of the dynamical mechanism of neural spikes between a single neuron and the networks behaviors.

\section{Acknowledgments}

This work was supported by the Key Project of National Natural Science Foundation of China (NSFC) (11232005) and was supported by the Fundamental Research Funds for the Central Universities of China.

\section{References}

[1] J. Anderson, I. Lampl, I. Reichova, M. Carandini, and D. Ferster, "Stimulus dependence of two-state fluctuations of membrane potential in cat visual cortex," Nature Neuroscience, vol. 3, no. 6, pp. 617-621, 2000.

[2] I. Lampl, I. Reichova, and D. Ferster, "Synchronous membrane potential fluctuations in neurons of the cat visual cortex," Neuron, vol. 22, no. 2, pp. 361-374, 1999.

[3] M. Steriade, A. Nunez, and F. Amzica, "Intracellular analysis of relations between the slow $(<1 \mathrm{~Hz})$ neocortical oscillation and other sleep rhythms of the electroencephalogram," The Journal of Neuroscience, vol. 13, no. 8, pp. 3266-3283, 1993.

[4] C. C. H. Petersen, T. T. G. Hahn, M. Mehta, A. Grinvald, and B. Sakmann, "Interaction of sensory responses with spontaneous depolarization in layer 2/3 barrel cortex," Proceedings of the National Academy of Sciences of the United States of America, vol. 100, no. 23, pp. 13638-13643, 2003.

[5] N. Parga and L. F. Abbott, "Network model of spontaneous activity exhibiting synchronous transitions between up and down states," Neuroscience, vol. 1, no. 1, pp. 57-66, 2007.

[6] I. Timofeev, F. Grenier, and M. Steriade, "Disfacilitation and active inhibition in the neocortex during the natural sleepwake cycle: an intracellular study," Proceedings of the National Academy of Sciences of the United States of America, vol. 98, no. 4, pp. 1924-1929, 2001.

[7] R. N. S. Sachdev, F. F. Ebner, and C. J. Wilson, "Effect of subthreshold up and down states on the whisker evoked response in somatosensory cortex," Journal of Neurophysiology, vol. 92, no. 6, pp. 3511-3521, 2004.

[8] B. Haider, A. Duque, A. R. Hasenstaub, Y. Yu, and D. A. McCormick, "Enhancement of visual responsiveness by spontaneous local network activity in vivo," Journal of Neurophysiology, vol. 97, no. 6, pp. 4186-4202, 2007.

[9] I. Timofeev, F. Grenier, M. Bazhenov, T. J. Sejnowski, and M. Steriade, "Origin of slow cortical oscillations in deafferented cortical slabs," Cerebral Cortex, vol. 10, no. 12, pp. 1185-1199, 2000 .

[10] F. B. Vialatte, J. Dauwels, M. Maurice, Y. Yamaguchi, and A. Cichocki, "On the synchrony of steady state visual evoked potentials and oscillatory burst events," Congnitive Neurodynamics, vol. 3, no. 3, pp. 251-261, 2009. 
[11] D. M. Alexander and C. van Leeuwen, "Mapping of contextual modulation in the population response of primary visual cortex," Congnitive Neurodynamics, vol. 4, no. 1, pp. 1-24, 2010.

[12] E. A. Stern, D. Jaeger, and C. J. Wilson, "Membrane potential synchrony of simultaneously recorded striatal spiny neurons in vivo," Nature, vol. 394, no. 6692, pp. 475-478, 1998.

[13] M. Brecht, M. Schneider, B. Sakmann, and T. W. Margrie, "Hisker movements evoked by stimulation of single pyramidal cells in rat motor cortex," Nature, vol. 427, pp. 704-710, 2004.

[14] R. Houweling and M. Brecht, "Behavioural report of single neuron stimulation in somatosensory cortex," Nature, vol. 451, no. 7174, pp. 65-68, 2008.

[15] C. T. Li, M. Poo, and Y. Dan, "Burst spiking of a single cortical neuron modifies global brain state," Science, vol. 324, no. 5927, pp. 643-646, 2009.

[16] R. Wang, Z. Zhang, and G. Chen, "Energy coding and energy functions for local activities of brain," Neurocomputing, vol. 73, no. 1-3, pp. 139-150, 2009.

[17] R. Wang, Z. Zhang, and G. Chen, "Energy function and energy evolution on neural population," IEEE Transactions on Neural Networks, vol. 19, no. 3, pp. 535-538, 2008.

[18] R. Wang and Z. Zhang, "Energy coding in biological neural network," Cognitive Neurodynamics, vol. 1, no. 3, pp. 203-212, 2007.

[19] Y. Liu, R. Wang, Z. Zhang, and X. Jiao, "Analysis on stability of neural network in the presence of inhibitory neurons," Congnitive Neurodynamics, vol. 4, no. 1, pp. 61-68, 2010.

[20] M. Steriade, D. McCormick, and T. Sejnowski, "Thalamocortical oscillations in the sleeping and aroused brain," Science, vol. 262, no. 5134, pp. 679-685, 1993.

[21] A. Destexhe, D. Contreras, and M. Steriade, "Spatiotemporal analysis of local field potentials and unit discharges in cat cerebral cortex during natural wake and sleep states," The Journal of Neuroscience, vol. 19, no. 11, pp. 4595-4608, 1999.

[22] D. Gervasoni, S.-C. Lin, S. Ribeiro, E. S. Soares, J. Pantoja, and M. A. L. Nicolelis, "Global forebrain dynamics predict rat behavioral states and their transitions," The Journal of Neuroscience, vol. 24, no. 49, pp. 11137-11147, 2004.

[23] J. Qu, R. Wang, Y. Du, and J. Cao, "Synchronization study ringlike and grid-like neuronal networks," Congnitive Neurodynamics, vol. 6, no. 1, pp. 21-31, 2012.

[24] X. Xu and R. Wang, "Neurodynamics of transitions between up and down states,". In press.

[25] M. Rubinov, O. Sporns, J.-P. Thivierge, and M. Breakspear, "Neurobiologically realistic determinants of self-organized criticality in networks of spiking neurons," PLoS Computational Biology, vol. 7, no. 6, Article ID e1002038, 2011.

[26] S. R. Williams, S. R. Christensen, G. J. Stuart, M. Häusser, and M. Membrane, "Potential bistability is controlled by the hyperpolarization activated current $\mathrm{I}(\mathrm{H})$ in rat cerebellar Purkinje neurons in vitro," The Journal of Physiology, vol. 1, part 2, no. 539, pp. 469-483, 2002.

[27] A. Roth and M. Häusser, "Compartmental models of rat cerebellar Purkinje cells based on simultaneous somatic and dendritic patchclamp recordings," The Journal of Physiology, vol. 535, pp. 445-472, 2001.

[28] R. Llinas and M. Sugimori, "Electrophysiological properties of in vitro Purkinje cell somata in mammalian cerebellar slices," The Journal of Physiology, vol. 305, pp. 171-195, 1980.

[29] R. Llinas and M. Sugimori, "Electrophysiological properties of in vitro Purkinje cell dendrites in mammalian cerebellar slices," The Journal of Physiology, vol. 305, pp. 197-213, 1980.
[30] Y. Loewenstein, S. Mahon, P. Chadderton et al., "Bistability of cerebellar Purkinje cells modulated by sensory stimulation," Neuroscience, vol. 8, no. 2, pp. 202-211, 2005. 


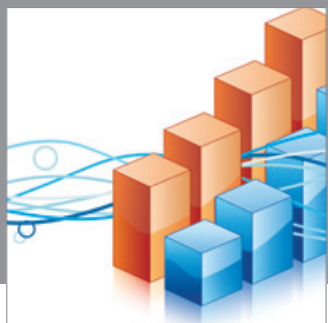

Advances in

Operations Research

mansans

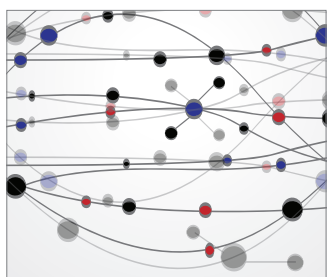

The Scientific World Journal
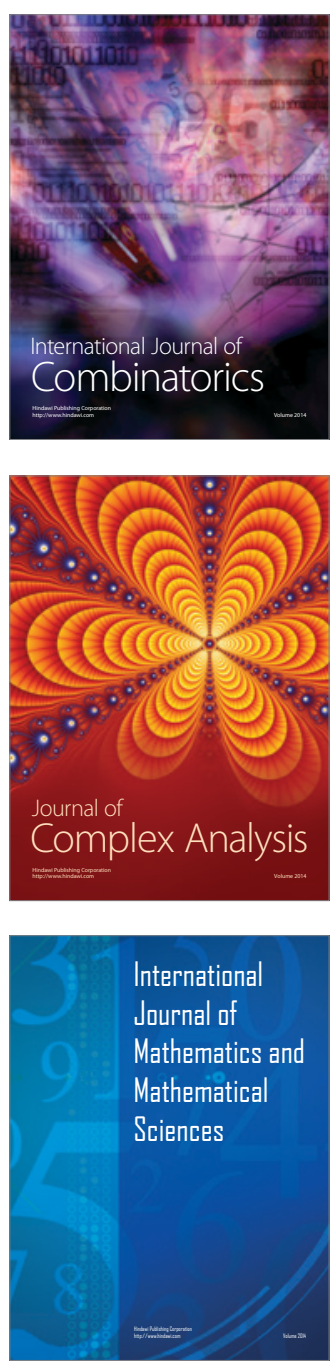
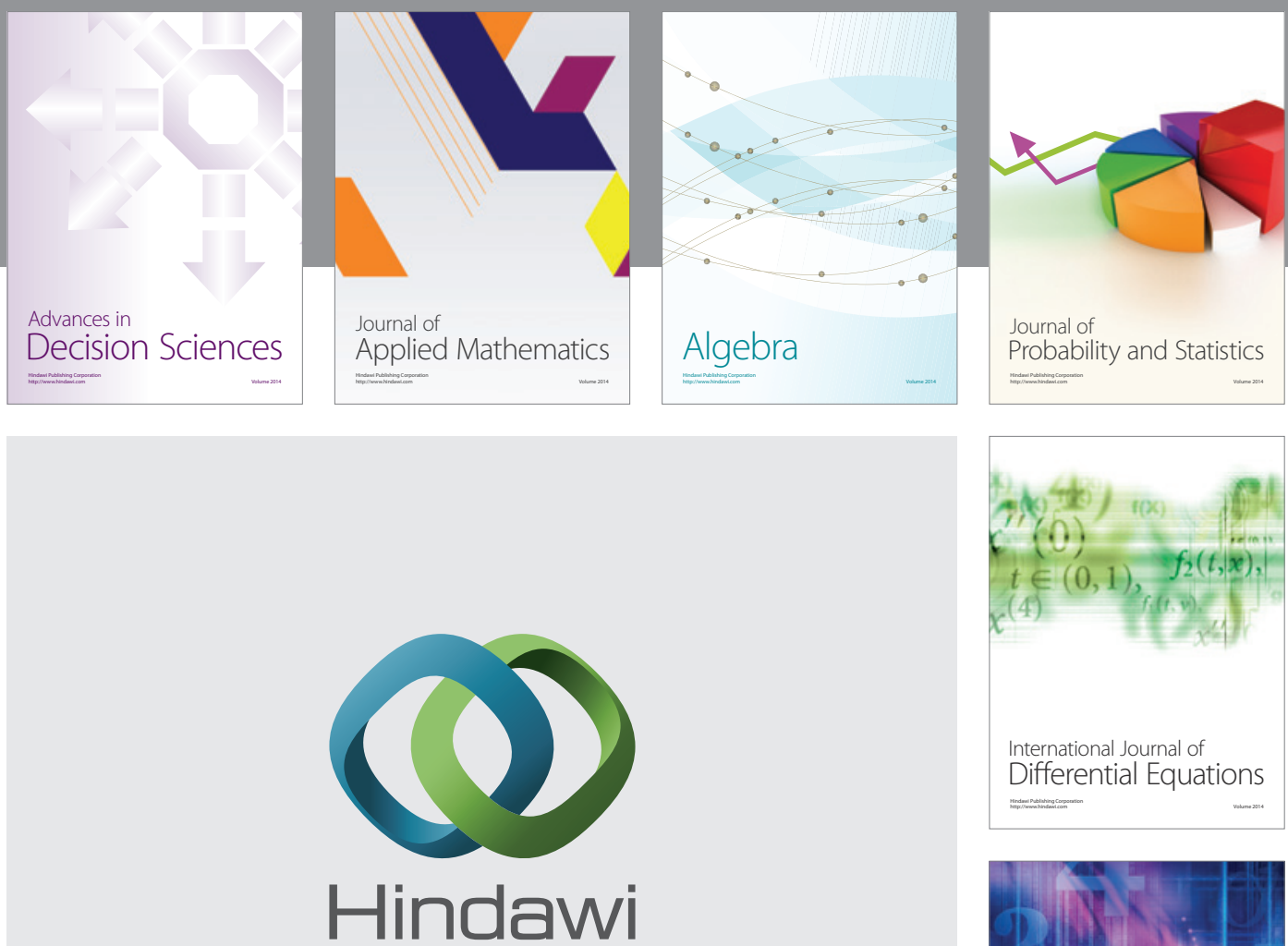

Submit your manuscripts at http://www.hindawi.com
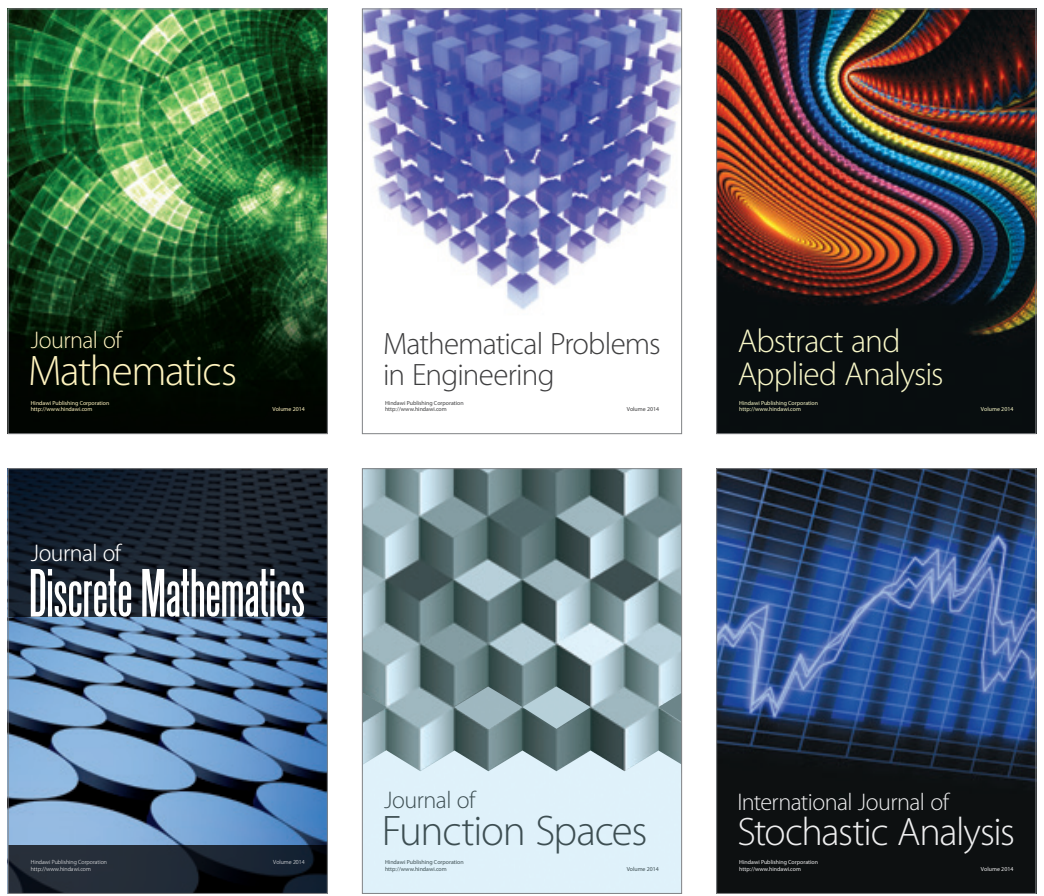

Journal of

Function Spaces

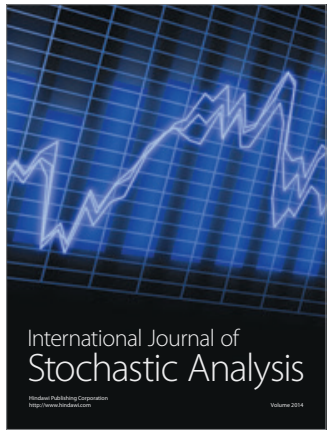

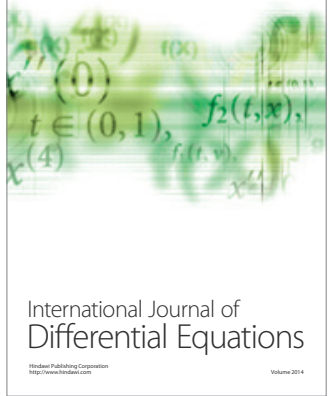
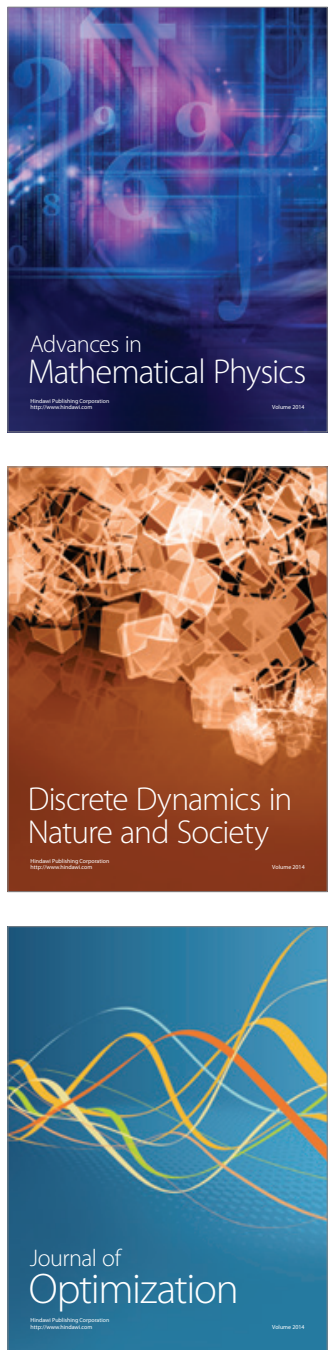